\title{
Thermodynamics of topological black holes in Brans-Dicke gravity with a power-law Maxwell field
}

\author{
M. Kord Zangeneh, ${ }^{1, *}$ M. H. Dehghani, ${ }^{1,2, \dagger}$ and A. Sheykhi ${ }^{1,2, \ddagger}$ \\ ${ }^{1}$ Physics Department and Biruni Observatory, College of Sciences, Shiraz University, Shiraz 71454, Iran \\ ${ }^{2}$ Research Institute for Astronomy and Astrophysics of Maragha (RIAAM), P.O. Box 55134-441, Maragha, Iran
}

\begin{abstract}
In this paper, we present a new class of higher-dimensional exact topological black hole solutions of the Brans-Dicke theory in the presence of a power-law Maxwell field as the matter source. For this aim, we introduce a conformal transformation which transforms the Einstein-dilaton-power-law Maxwell gravity Lagrangian to the Brans-Dicke-power-law Maxwell theory one. Then, by using this conformal transformation, we obtain the desired solutions. Next, we study the properties of the solutions and conditions under which we have black holes. Interestingly enough, we show that there is a cosmological horizon in the presence of a negative cosmological constant. Finally, we calculate the temperature and charge and then by calculating the Euclidean action, we obtain the mass, the entropy and the electromagnetic potential energy. We find that the entropy does not respect the area law, and also the conserved and thermodynamic quantities are invariant under conformal transformation. Using these thermodynamic and conserved quantities, we show that the first law of black hole thermodynamics is satisfied on the horizon.
\end{abstract}

PACS numbers:

\section{INTRODUCTION}

During the years between 1905 and 1915, many attempts were done to enter gravity into special relativity. Einstein, himself, and Nordstrom were pioneers in this issue. Nordstrom presented a scalar theory of gravitation [1] while Einstein proposed a tensorial theory of gravitation [2]. Eventually, the Einstein tensorial theory of gravitation, known today as general relativity (GR), was more successful to pass observational tests and became the standard theory of gravitation [3]. However, some time later, GR showed some failures. The most important failure was the inability to describe accelerating expansion of the Universe [4]. In addition to latter failure, the fact that Mach's principle is not respected by GR led physicists to modify Einstein theory of gravity. One of the most impressive and physically viable modifications is Brans-Dicke (BD) theory adding a scalar degree of freedom to previous 10 degrees of freedom coming from metric tensor $g_{\mu \nu}$ in four dimensions [5]. This theory was motivated from one side by Mach's principle encoded by varying gravitational constant in it and from other side by Dirac's large number hypothesis. These two motivations are summed up in this theory by considering a scalar field, $\Phi$, which is inversely proportional to the gravitational constant $G$ and nonminimally coupled to gravitation [6]. Another important aspect of BD theory is its equivalence to several other modifications of GR in particular $f(R)$ theories that causes better understating of these theories [7]. Also, BD theory may be considered as a special case of more general scalar-tensor gravity

\footnotetext{
*Electronic address: mkzangeneh@shirazu.ac.ir

$\dagger$ Electronic address: mhd@shirazu.ac.ir

‡Electronic address: asheykhi@shirazu.ac.ir
}

which has been considered in [8].

The first solutions of BD theory have been published by Brans himself. These solutions were in four dimensions and classified in four classes [9]. Among these four classes just two of them are really independent [10] while just one of these two independent classes are valid for all values of $\omega$. Also, linearly charged solutions of BD gravity have been presented in [11]. These latter solutions are just allowed in the presence of a trivial (constant) scalar field in four dimensions. This is due to the conformal invariance of linear Maxwell Lagrangian in four dimensions. Since linear Maxwell Lagrangian is no longer conformally invariant in higher dimensions, the linear Maxwell field can play the role of source of scalar field for higher-dimensional BD gravity [11]. The highly nonlinear nature of BD theory makes it non-straightforward in many cases to find the solutions by solving BD field equations directly. Fortunately, there is a way to overcome this problem. As it has been shown in many cases, the solutions of BD theory can be found by applying a conformal transformation on known solutions of other theories such as dilaton theory [12]. For instance, linearly charged rotating black branes have been obtained in BD theory by applying a conformal transformation from the known solutions of dilaton gravity [13]. Also, by using a similar method, asymptotic anti-de Sitter (AdS) black holes and topological black holes with nonflat and nonAdS asymptotic behaviors have been investigated in Refs. [14] and [15], respectively. The fact that one can transform the Lagrangian of BD gravity to the Lagrangian of dilaton gravity by a conformal transformation has been also used for more general scalar-tensor gravities in [8] to investigate the different aspects of scalar-tensor gravities in the presence of nonlinear electromagnetic field [8].

The nonlinear electrodynamics was first introduced by Born and Infeld in order to obtain the finite energy density for an electron [16]. In recent years other types 
of Born-Infeld like nonlinear electrodynamics have been proposed $[17,18]$. The studies were also extended to dilaton gravity [19]. However, the energy-momentum tensors of Born-Infeld theory or the Born-Infeld like theories are not traceless even in four dimensions. A nonlinear electrodynamic theory with a traceless energymomentum tensor in higher dimensions has been introduced in [20]. The power-law Maxwell matter source is conformal invariant in higher dimensions for special choice of power. To be more clear, the Lagrangian of power-law Maxwell field $\left(-F_{\mu \nu} F^{\mu \nu}\right)^{p}$ is invariant under conformal transformation $g_{\mu \nu} \rightarrow \Omega^{2} g_{\mu \nu}, A_{\mu} \rightarrow A_{\mu}$ provided $p=(n+1) / 4$. Despite of the mentioned special property for $p=(n+1) / 4$, many solutions have been studied from different aspects with nonfixed $p$ [21]. In this paper we extend the study of [15] to nonlinear powerlaw Maxwell field electrodynamics and try to solve field equations of BD theory by using known solutions of dilaton gravity that we have recently presented in [22].

The layout of the paper is as follows. In the next section, we introduce the basic field equations of BD theory with power-law Maxwell Lagrangian. We also introduce a conformal transformation which transforms this theory to Einstein-dilaton gravity. In Sec. III, we obtain a class of topological black hole solution of this theory and investigate its properties. In Sec. IV, we investigate thermodynamics of the solutions and check the validity of the first law of thermodynamics. We finish our paper with closing remarks in the last section.

\section{FIELD EQUATIONS AND CONFORMAL TRANSFORMATION}

The action of Brans-Dicke theory coupled to a powerlaw Maxwell (BDPM) field can be written as

$$
\begin{array}{r}
S_{B D}=-\frac{1}{16 \pi} \int_{\mathcal{M}} d^{n+1} x \sqrt{-g}\left(\Phi \mathcal{R}-\frac{\omega}{\Phi}(\nabla \Phi)^{2}\right. \\
\left.-V(\Phi)+(-F)^{p}\right)
\end{array}
$$

where $\mathcal{R}$ is the Ricci scalar, $\omega$ is the coupling constant, $\Phi$ denotes the $\mathrm{BD}$ scalar field, $p$ is a constant determining the nonlinearity of the electromagnetic field and $V(\Phi)$ is a self-interacting potential for $\Phi$. In Eq. (1), $F=F_{\mu \nu} F^{\mu \nu}$ where $F_{\mu \nu}=\partial_{[\mu} A_{\nu]}$ is the electromagnetic tensor field and $A_{\mu}$ is the vector potential. Varying the action (1) with respect to the metric $g_{\mu \nu}$, the scalar field $\Phi$ and the electromagnetic field $A_{\mu}$, one can obtain the field equations as

$$
\begin{aligned}
G_{\mu \nu}= & \frac{\omega}{\Phi^{2}}\left(\nabla_{\mu} \Phi \nabla_{\nu} \Phi-\frac{1}{2} g_{\mu \nu}(\nabla \Phi)^{2}\right) \\
& +\frac{1}{\Phi}\left(\nabla_{\mu} \nabla_{\nu} \Phi-g_{\mu \nu} \nabla^{2} \Phi\right) \\
& -\frac{V(\Phi)}{2 \Phi} g_{\mu \nu}-\frac{2(-F)^{p-1}}{\Phi}\left(\frac{1}{4} F g_{\mu \nu}-p F_{\mu \lambda} F_{\nu}{ }^{\lambda}\right), \\
\nabla^{2} \Phi= & \frac{(n-4 p+1)(-F)^{p}}{2[(n-1) \omega+n]} \\
& +\frac{\left[(n-1) \Phi \frac{d V(\Phi)}{d \Phi}-(n+1) V(\Phi)\right]}{2[(n-1) \omega+n)]} \\
& \nabla_{\mu}\left[(-F)^{p-1} F^{\mu \nu}\right]=0,
\end{aligned}
$$

As it is clear from the right-hand side of (2), there are second order derivatives of the scalar field. This fact makes the problem of solving the field equations (2)-(4) difficult. Fortunately, this difficulty can be circumvented by using the following conformal transformation:

$$
\begin{gathered}
\tilde{\Phi}=\frac{\sqrt{n+\omega(n-1)}}{2} \ln \Phi, \\
\tilde{g}_{\mu \nu}=\Phi^{2 /(n-1)} g_{\mu \nu}, \\
F_{\mu \nu}=\tilde{F}_{\mu \nu},
\end{gathered}
$$

where $\tilde{\Phi}$ is the dilaton field and

$$
\tilde{V}(\tilde{\Phi})=\Phi^{-(n+1) /(n-1)} V(\Phi) .
$$

Indeed, the transformation (5) transforms the action (1) to the action of Einstein-dilaton gravity coupled to power-law Maxwell field [22]

$$
\begin{aligned}
S_{E D}= & -\frac{1}{16 \pi} \int d^{n+1} x \sqrt{-\tilde{g}}\left\{\tilde{\mathcal{R}}-\frac{4}{n-1}(\tilde{\nabla} \tilde{\Phi})^{2}\right. \\
& \left.-\tilde{V}(\tilde{\Phi})+\left(-e^{-4 \alpha \tilde{\Phi} /(n-1)} \tilde{F}\right)^{p}\right\},
\end{aligned}
$$

where $\alpha=(n-4 p+1) / 2 p \sqrt{n+\omega(n-1)}$. One may note that $\alpha$ is a constant which determines the strength of coupling of the scalar and electromagnetic field and is equal to zero for $p=(n+1) / 4$. So, we assume that $p \neq(n-1) / 4$. The field equations corresponding to action (7) are

$$
\begin{aligned}
\tilde{\mathcal{R}}_{\mu \nu}= & \tilde{g}_{\mu \nu}\left\{\frac{\tilde{V}(\tilde{\Phi})}{n-1}+\frac{(2 p-1)}{n-1}\left(-e^{-4 \alpha \tilde{\Phi} /(n-1)} \tilde{F}\right)^{p}\right\} \\
& +\frac{4}{n-1} \partial_{\mu} \tilde{\Phi} \partial_{\nu} \tilde{\Phi}+2 p e^{-4 \alpha p \tilde{\Phi} /(n-1)}(-\tilde{F})^{p-1} \tilde{F}_{\mu \lambda} \tilde{F}_{\nu}{ }^{\lambda},
\end{aligned}
$$

$$
\begin{aligned}
\tilde{\nabla}^{2} \tilde{\Phi}-\frac{n-1}{8} \frac{d \tilde{V}(\tilde{\Phi})}{d \tilde{\Phi}}-\frac{p \alpha}{2} e^{-4 \alpha p \tilde{\Phi} /(n-1)}(-\tilde{F})^{p} & =0,(9) \\
\tilde{\nabla}_{\mu}\left(e^{-4 \alpha p \tilde{\Phi} /(n-1)}(-\tilde{F})^{p-1} \tilde{F}^{\mu \nu}\right) & =(10)
\end{aligned}
$$


One should note that the field equation (8) does not contain second order derivatives of scalar field and, therefore, one can solve Eqs. (8)-(9) in a simpler way than the field equations (2)-(4). In [22], the solution of Einsteindilaton-power Maxwell (EDPM) gravity has been presented. We first review these solutions in the next section.

\section{TOPOLOGICAL BLACK HOLE SOLUTIONS IN BDPM GRAVITY}

In order to obtain the topological black hole solutions of BDPM gravity, we first review the $(n+1)$-dimensional topological black hole solutions of EDPM gravity (7) presented in [22]. The metric of a general static spacetime can be written as

$$
d \tilde{s}^{2}=-f(r) d t^{2}+\frac{d r^{2}}{f(r)}+r^{2} R^{2}(r) d \Omega_{n-1}^{2},
$$

where $d \Omega_{n-1}^{2}=h_{i j}(x) d x^{i} d x^{j}$ is the metric of an $(n-1)$ dimensional constant curvature hypersurface. The curvature of this hypersurface is equal to $(n-1)(n-2) k$, where $k=0, \pm 1$. We have shown in [22] that in order to have topological black holes with a general $k$ and $p$, the potential should be chosen as

$$
\tilde{V}(\tilde{\Phi})=2 \Lambda_{1} e^{2 \zeta_{1} \tilde{\Phi}}+2 \Lambda_{2} e^{2 \zeta_{2} \tilde{\Phi}}+2 \Lambda e^{2 \zeta_{3} \tilde{\Phi}}
$$

where

$$
\begin{gathered}
\zeta_{1}=\frac{2}{(n-1) \alpha}, \quad \zeta_{2}=\frac{2 p\left(n-1+\alpha^{2}\right)}{(n-1)(2 p-1) \alpha} \\
\zeta_{3}=\frac{2 \alpha}{n-1}, \quad \Lambda_{1}=\frac{k(n-1)(n-2) \alpha^{2}}{2 b^{2}\left(\alpha^{2}-1\right)} \\
\Lambda_{2}=\frac{2^{p-1}(2 p-1)(p-1) \alpha^{2} q^{2} p}{\Pi b^{\frac{2(n-1) p}{2 p-1}}} .
\end{gathered}
$$

The solutions of EDPM field equations are [22]

$$
\begin{gathered}
f(r)=\frac{k(n-2)\left(1+\alpha^{2}\right)^{2} r^{2 \gamma}-\frac{m}{r^{(n-1)(1-\gamma)-1}}}{\left(1-\alpha^{2}\right)\left(\alpha^{2}+n-2\right) b^{2 \gamma}}-\frac{2 \Lambda b^{2 \gamma}\left(1+\alpha^{2}\right)^{2} r^{2(1-\gamma)}}{(n-1)\left(n-\alpha^{2}\right)} \\
+\frac{\hat{q}^{2 p}}{r^{\Upsilon+(n-1)(1-\gamma)-1}} \\
\tilde{\Phi}(r)=\frac{(n-1) \alpha}{2\left(\alpha^{2}+1\right)} \ln \left(\frac{b}{r}\right) \\
R(r)=\left(\frac{b}{r}\right)^{\gamma}
\end{gathered}
$$

$$
\tilde{A}_{t}=\frac{q b^{\frac{(2 p+1-n) \gamma}{(2 p-1)}}}{\Upsilon r^{\Upsilon}}
$$

where

$$
\begin{aligned}
\hat{q}^{2 p} & =\frac{2^{p} p(2 p-1) q^{2 p}}{(1-\gamma) \Pi \Upsilon b^{2(n-2) p \gamma /(2 p-1)}}, \\
\Pi & =\alpha^{2}+\left(n-1-\alpha^{2}\right) p \\
\Upsilon & =\frac{\left(n-2 p+\alpha^{2}\right)}{(2 p-1)\left(1+\alpha^{2}\right)}
\end{aligned}
$$

$b$ is an arbitrary nonzero positive constant, and $\gamma=$ $\alpha^{2} /\left(\alpha^{2}+1\right)$. In the above relations $m$ and $q$ are two constants proportional to the mass and charge of the black holes, respectively, and $\Lambda$ is a free constant which can be interpreted as cosmological constant, since in the absence of the dilaton field $(\alpha=0)$, we have $V(\Phi)=2 \Lambda$. Thus as usual, we redefine it as $\Lambda=-n(n-1) / 2 l^{2}$ where $l$ is a constant with length dimension.

With the solutions of EDPM gravity in hand, we can construct $(n+1)$-dimensional solutions of BDPM by applying conformal transformation (5). The line element of the spacetime can be obtained as

$$
d s^{2}=-U(r) d t^{2}+\frac{d r^{2}}{V(r)}+r^{2} H^{2}(r) h_{i j} d x^{i} d x^{j},
$$

where $U(r), V(r), H(r)$ and $\Phi(r)$ are

$$
\begin{aligned}
U(r)= & \left(\frac{b}{r}\right)^{-\Gamma} f(r) \\
= & \frac{k(n-2)\left(1+\alpha^{2}\right)^{2}}{\left(1-\alpha^{2}\right)\left(\alpha^{2}+n-2\right)}\left(\frac{r}{b}\right)^{\Gamma+2 \gamma} \\
& -\frac{m b^{-\Gamma}}{r^{-\Gamma+(n-1)(1-\gamma)-1}}+\frac{b^{-\Gamma} \hat{q}^{2 p}}{r^{-\Gamma+\Upsilon+(n-1)(1-\gamma)-1}} \\
& -\frac{2 \Lambda b^{2}\left(1+\alpha^{2}\right)^{2}}{(n-1)\left(n-\alpha^{2}\right)}\left(\frac{r}{b}\right)^{\Gamma+2(1-\gamma)}
\end{aligned}
$$

$$
\begin{aligned}
V(r)= & \left(\frac{b}{r}\right)^{\Gamma} f(r) \\
= & \frac{k(n-2)\left(1+\alpha^{2}\right)^{2}}{\left(1-\alpha^{2}\right)\left(\alpha^{2}+n-2\right)}\left(\frac{r}{b}\right)^{-\Gamma+2 \gamma} \\
& -\frac{m b^{\Gamma}}{r^{\Gamma+(n-1)(1-\gamma)-1}}+\frac{b^{\Gamma} \hat{q}^{2 p}}{r^{\Gamma+\Upsilon+(n-1)(1-\gamma)-1}} \\
& -\frac{2 \Lambda b^{2}\left(1+\alpha^{2}\right)^{2}}{(n-1)\left(n-\alpha^{2}\right)}\left(\frac{r}{b}\right)^{-\Gamma+2(1-\gamma)}
\end{aligned}
$$


where $\Gamma=4 p \gamma /(n-4 p+1)$. The electromagnetic gauge potential $A_{t}$ and potential of scalar field can also be obtained as

$$
\begin{gathered}
A_{t}=\frac{q b^{\frac{(2 p+1-n) \gamma}{(2 p-1)}}}{\Upsilon r^{\Upsilon}}, \\
V(\Phi)=2 \Lambda_{1} \Phi^{\xi_{1}}+2 \Lambda_{2} \Phi^{\xi_{2}}+2 \Lambda \Phi^{\xi_{3}},
\end{gathered}
$$

where

$$
\begin{gathered}
\xi_{1}=\frac{n-4 p+1+p \alpha^{2}(n+1)}{p(n-1) \alpha^{2}}, \quad \xi_{2}=\frac{n-4 p+1+2 p \alpha^{2}}{\alpha^{2}(2 p-1)}, \\
\xi_{3}=\frac{p(n-3)+n+1}{p(n-1)} .
\end{gathered}
$$

It is worth mentioning that for the case of $p=1$ and $k=0$, both $\Lambda_{1}$ and $\Lambda_{2}$ vanish and therefore $V(\Phi)=2$, $\Lambda \Phi^{2}$ [13]. Also, one may note that as $\omega \rightarrow \infty(\alpha=\gamma=0)$ for the linear Maxwell theory $(p=1)$, solutions (19) and (20) reduce to

$$
U(r)=V(r)=k-\frac{m}{r^{n-2}}+\frac{2 q^{2}}{(n-1)(n-2) r^{2(n-2)}}-\frac{2 \Lambda}{n(n-1)} r^{2},
$$

which describes an $(n+1)$-dimensional asymptotically (A)dS topological black hole for positive (negative) cosmological constant with a flat $(k=0)$, spherical $(k=1)$ or hyperbolic $(k=-1)$ horizons (see for example [23$25]$ ). For $p=1$, our solutions reproduce the solutions of Ref. [15].

One should note that there are some constraints on the values of $p$ and $\alpha$. We stress on this fact that the electromagnetic gauge potential $A_{t}$ should vanish at infinity and, therefore, $\Upsilon>0$ which leads to

$$
\frac{1}{2}<p<\frac{n+\alpha^{2}}{2} .
$$

On the other hand, we assume that the scalar field of $\mathrm{BD}$ be a localized function. That is $\Phi$ should go to zero as $r \rightarrow \infty$ which implies $\Gamma>0$ and therefore

$$
p<\frac{n+1}{4} .
$$

One can summarize (26) and (27) to receive

$$
\frac{1}{2}<p<\frac{n+1}{4},
$$

which is the allowed range of $p$. In the above allowed range of $p$ the charge term of the metric functions $U(r)$ given in Eq. (19) dominates in the vicinity of $r=0$. Consequently, it is sufficient to check the sign of the power of $r$ in the mass term in $U(r)$ in order to guarantee that the

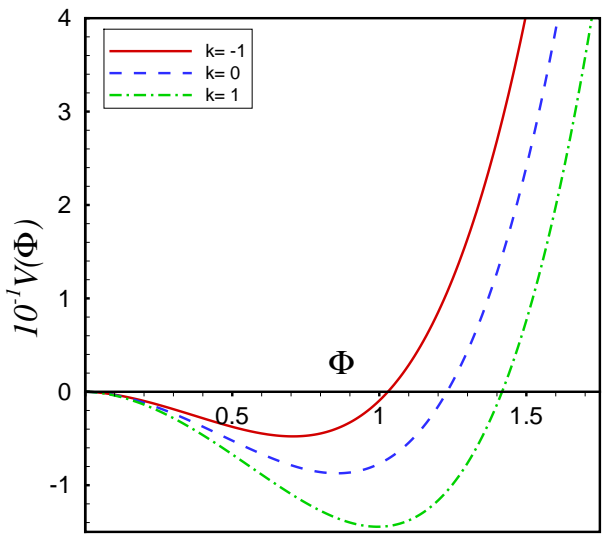

FIG. 1: The self-interacting potential $10^{-1} V(\Phi)$ versus $\Phi$ for $n=5, p=1.2, \alpha=0.6, l=b=1, \Lambda=-10$ and $q=10$.

effects of mass and charge vanish at infinity in both $U(r)$ and $V(r)$ given by (19) and (20) [note that according to (28), $\Gamma>0$ ]. One can easily show that the effects of mass in $U(r)$ disappear at infinity provided

$$
\alpha^{2}<\frac{(n-4 p+1)(n-2)}{n+1} .
$$

or equivalently

$$
\omega>-\frac{n}{n-1}+\frac{(n-4 p+1)(n+1)}{4 p^{2}(n-1)(n-2)} .
$$

Hence the allowed ranges of $p$ and $\alpha$ or $\omega$ are

$$
\frac{1}{2}<p<\frac{n+1}{4}, \quad \alpha^{2}<\frac{(n-4 p+1)(n-2)}{n+1}
$$

or equivalently

$$
\frac{1}{2}<p<\frac{n+1}{4}, \quad \omega>-\frac{n}{n-1}+\frac{(n-4 p+1)(n+1)}{4 p^{2}(n-1)(n-2)} .
$$

Before discussing the properties of our solutions, we pause to consider the stability of the system. In order to have a stable system, it is necessary for $V(\Phi)$ to have a lower bound. As it can be seen from Fig. $1, V(\Phi)$ is bounded from below for suitable choices of the parameters.

\section{A. Properties of the solutions}

Now, we are ready to discuss physical properties of the topological BD black holes in the presence of power-law Maxwell field. First, we discuss the domain of validity of our solutions. It is worth mentioning that, as one can see from Eqs. (19) and (20), our solutions are ill defined for the string case where $\alpha=1$ [corresponding to the 
case of $\left.\omega=\left[(n-4 p+1)^{2}-4 n p^{2}\right] / 4 p^{2}(n-1)\right]$, except for the flat horizon case i.e. $k=0$. For other values of $\alpha$, our solutions are well defined in the permitted ranges of $p$ and $\alpha$. Second, it is easy to show that there is an essential singularity at $r=0$ since the Kretschmann scalar $R_{\mu \nu \lambda \sigma} R^{\mu \nu \lambda \sigma}$ diverges at $r=0$. Kretschmann scalar also is finite for $r \neq 0$ and vanishes as $r \rightarrow \infty$. Third, the charge term in both $V(r)$ and $U(r)$ is the dominant term in the vicinity of $r=0$ and goes to infinity for the allowed ranges of $p$ and $\alpha\left(\hat{q}^{2 p}>0\right)$. Thus, the singularity is timelike as in the case of Reissner-Nordstrom black holes. That is, there is no Schwarzschild-like solution and one encounters with the solutions with two inner and outer horizons, extreme black holes and naked singularities depending on the values of the metric parameters such as $p, q, m, \alpha$ and $k$. Fourth, we investigate the asymptotic behavior of the solutions. In order to consider the asymptotic behavior of our solutions, it is enough to investigate the behavior of the metric function $V(r)$ at infinity. In the case of $k=0$, for $\alpha^{2}<\min [(n-4 p+1) / 2 p,(n-4 p+1)(n-2) /(n+1)]$ [Note that $\alpha^{2}<(n-4 p+1) / 2 p$ implies that $-\Gamma+2(1-\gamma)>0$ and $\alpha^{2}<(n-4 p+1)(n-2) /(n+1)$ is $\left.(29)\right]$, the metric functions go to infinity as $r$ goes to infinity provided $\Lambda<0$, and one has cosmological horizon for $\Lambda>0$. In the case of $k= \pm 1$, for $\alpha^{2}>1$, the first term of $V(r)$ is the dominant term at infinity. Therefore, the metric functions go to infinity in the case of $k=-1$ and one has cosmological horizon in the case of $k=1$ provided $p<(n+1) / 6(-\Gamma+2 \gamma>0)$. For $\alpha^{2}<1$ where the fourth term of $V(r)$ is dominant at infinity, the metric functions go to infinity for $\Lambda<0$ and the solutions have cosmological horizon for $\Lambda>0$ provided $\alpha^{2}<\min [(n-4 p+1) / 2 p,(n-4 p+1)(n-2) /(n+1)]$.

Finally, we investigate the causal structure of our solutions. In order to do this, and find out whether the singularity is naked or not, we should study the zeros of $g^{r r}=V(r)$. Although it is difficult to find the roots of $V(r)=0$ analytically, we can gain some insight into the behavior of it by studying $m\left(r_{h}\right)$ :

$$
\begin{aligned}
m\left(r_{h}\right)= & \frac{k(n-2)\left(1+\alpha^{2}\right)^{2} b^{-2 \gamma}}{\left(1-\alpha^{2}\right)\left(\alpha^{2}+n-2\right)} r_{h}^{(n-3)(1-\gamma)+1} \\
& +\frac{\hat{q}^{2 p}}{r_{h}^{\Upsilon}}-\frac{2 \Lambda b^{2 \gamma}\left(1+\alpha^{2}\right)^{2}}{(n-1)\left(n-\alpha^{2}\right)} r_{h}^{(n+1)(1-\gamma)-1}
\end{aligned}
$$

which comes from this fact that $V\left(r_{h}\right)=0$ where $r_{h}$ is the radius of the horizon. We consider $m\left(r_{h}\right)$ in the absence of the cosmological horizon and with cosmological horizon, separately. In the absence of cosmological horizon, as $r_{h}$ goes from zero to infinity, the function $m\left(r_{h}\right)$ starts from infinity and goes to infinity as one can see in Figs. 2 and 3. The intersections of the line $m=$ constant with the curve $m\left(r_{h}\right)$ are inner and outer horizons $r_{-}$and $r_{+}$in these figures. There is also a minimum in these figures. The value of the minimum is $m_{\text {ext }}$ which is the

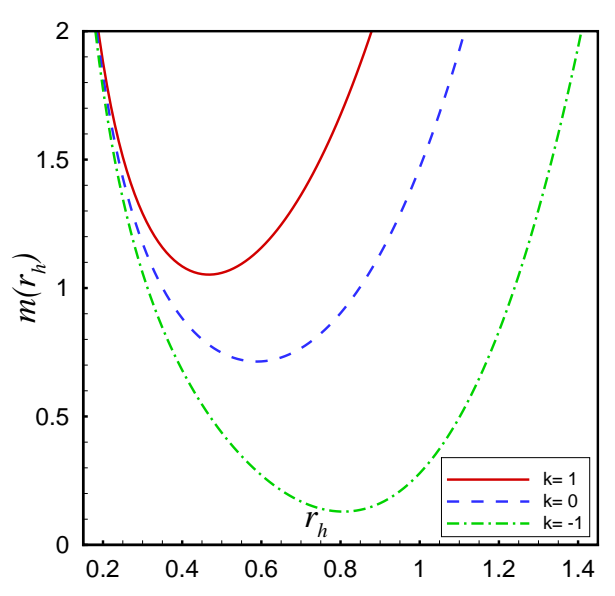

FIG. 2: The function $m\left(r_{h}\right)$ versus $r_{h}$ for $n=4, p=1.2$, $\alpha=0.26, l=b=1, \Lambda=-6$ and $q=0.6$.

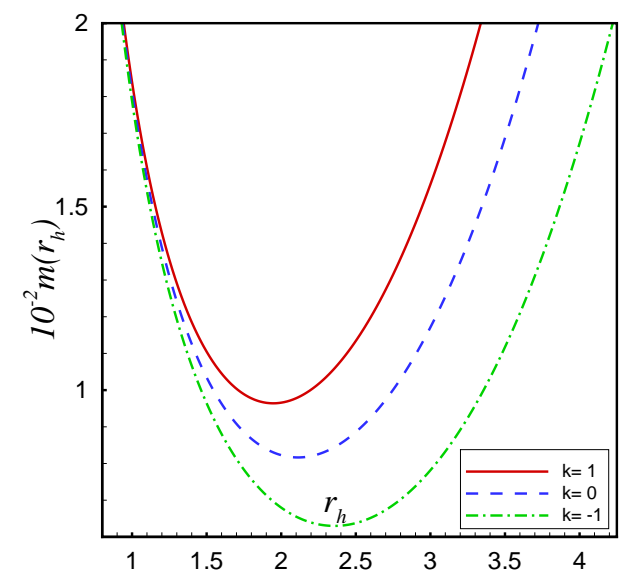

FIG. 3: The function $10^{-2} m\left(r_{h}\right)$ versus $r_{h}$ for $n=5, p=1.2$, $\alpha=0.6, l=b=1, \Lambda=-10$ and $q=10$.

solution of $V(r)=0=V^{\prime}(r)$ :

$$
\begin{aligned}
m_{\mathrm{ext}}= & \frac{2\left(\alpha^{2}+1\right)}{(2 p-1) \Upsilon} r_{\mathrm{ext}}^{(n+1)(1-\gamma)-1} \\
& \times\left[-\frac{2 \Lambda b^{2 \gamma} \Pi}{\left(n-\alpha^{2}\right)(n-1)}\right. \\
& \left.+\frac{k\left(\left(n-3+\alpha^{2}\right) p+1\right)(n-2)}{b^{2 \gamma}\left(1-\alpha^{2}\right)\left(\alpha^{2}+n-2\right)} r_{\mathrm{ext}}^{4 \gamma-2}\right]
\end{aligned}
$$

Thus, our solutions present black holes with inner and outer horizons located at $r_{-}$and $r_{+}$provided $m>m_{\text {ext }}$, an extreme black hole if $m=m_{\text {ext }}$ and a naked singularity provided $m<m_{\text {ext }}$ (See Figs. 4-7). For solutions with cosmological horizon, the function $m\left(r_{h}\right)$ starts from infinity and goes to minus infinity as $r_{h}$ increases from zero to infinity. In this case it is better to study the behavior 


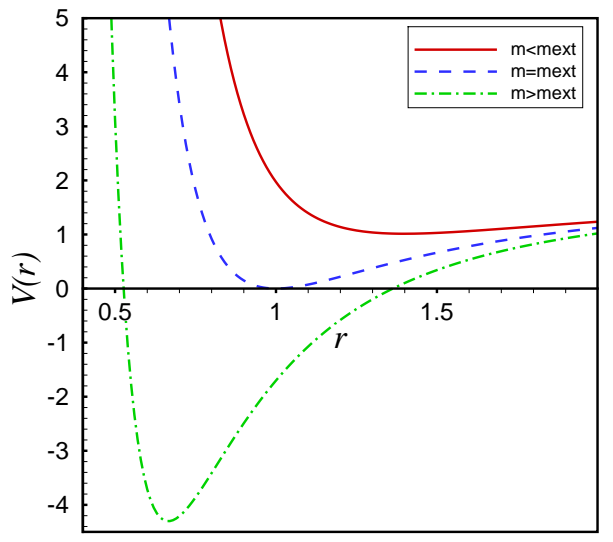

FIG. 4: The function $V(r)$ versus $r$ for $k=0, n=4, p=1.2$, $\alpha=0.26, l=b=1, \Lambda=-6$ and $r_{\text {ext }}=1$.

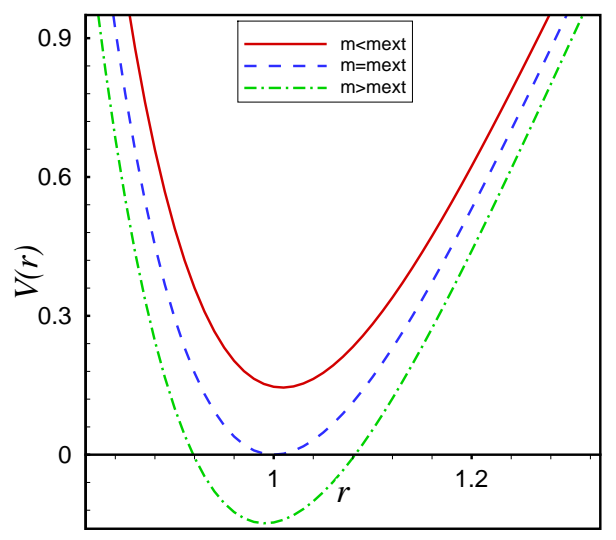

FIG. 5: The function $V(r)$ versus $r$ for $k=-1, n=6, p=1.1$, $\alpha=1.18, l=b=1, \Lambda=15$ and $r_{\text {ext }}=1$.

of $\partial m / \partial r_{h}$ which is given as

$$
\begin{aligned}
\frac{\partial m}{\partial r_{h}}= & \frac{k\left(\alpha^{2}+1\right)(n-2)}{\left(1-\alpha^{2}\right) b^{2 \gamma}} r_{h}^{(n-3)(1-\gamma)} \\
& -\frac{\Upsilon \hat{q}^{2 p}}{r_{h}^{\Upsilon+1}}-\frac{2 \Lambda\left(\alpha^{2}+1\right) b^{2 \gamma}}{(n-1)} r_{h}^{(n+1)(1-\gamma)-2}
\end{aligned}
$$

As it is clear from Eq. (35), $\partial m / \partial r_{h}$ has no zero for $k /\left(1-\alpha^{2}\right) \leq 0$ and $\Lambda>0$ and the function $m\left(r_{h}\right)$ starts at infinity and goes to minus infinity without any minimum or maximum. For this case our solution presents a naked singularity with cosmological horizon as Fig. 8 shows. When $\partial m / \partial r_{h}$ has zeros, the function $m\left(r_{h}\right)$ starts at infinity and goes to minus infinity with a minimum and a maximum as one can see in Fig. 9. In this case we have black holes with three inner, outer and cosmological horizons provided $m_{\text {ext }}<m<m_{\text {crit }}$ an extreme black hole with cosmological horizon if $m_{\mathrm{ext}}=m<$

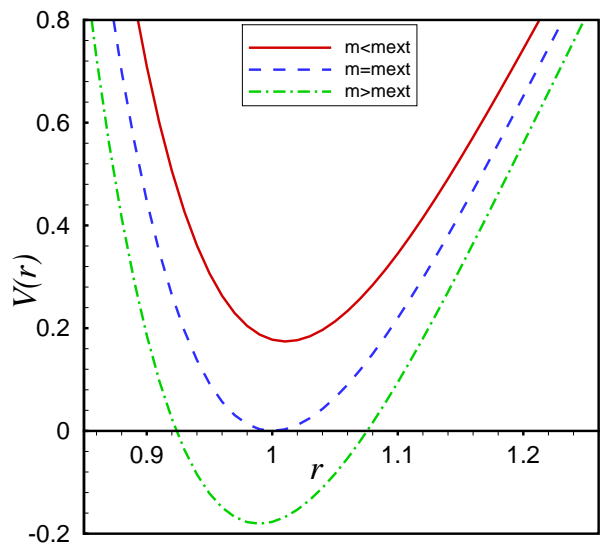

FIG. 6: The function $V(r)$ versus $r$ for $k=1, n=5, p=1.2$, $\alpha=0.63, l=b=1, \Lambda=-10$ and $r_{\text {ext }}=1$.

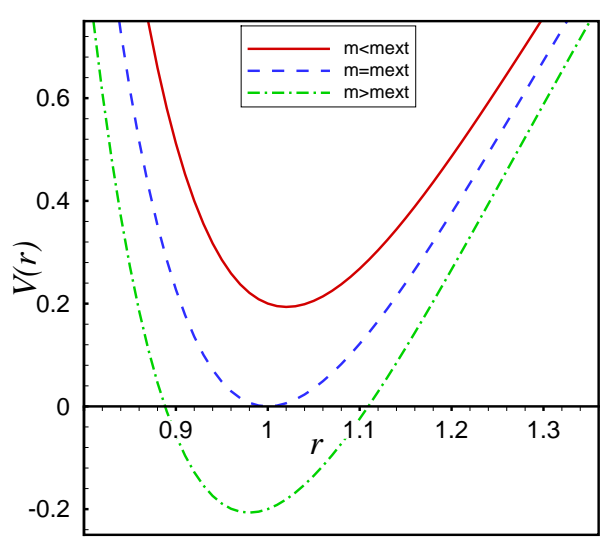

FIG. 7: The function $V(r)$ versus $r$ for $k=-1, n=5, p=1.2$, $\alpha=0.63, l=1, b=1.5, \Lambda=-10$ and $r_{\text {ext }}=1$.

$m_{\text {crit }}$ and a naked singularity with cosmological horizon for the cases $m<m_{\text {ext }}$ and $m \geq m_{\text {crit }}$ as one may see in Fig. 10. As it is clear from Figs. 9 and 10, we have cosmological horizon for $\Lambda<0$. This result is interesting since it shows that we can have cosmological horizon in the presence of a negative cosmological constant.

\section{THERMODYNAMICS OF TOPOLOGICAL BD BLACK HOLES}

In this section, we are going to check the validity of the first law of thermodynamics for the topological BD black holes with power-law Maxwell field. In order to do this, we should calculate the conserved and thermodynamic quantities. We start with temperature. The Hawking temperature of the topological black holes on the outer 


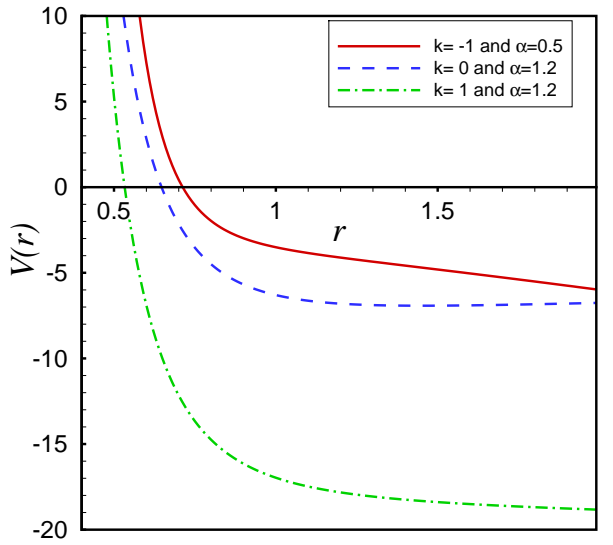

FIG. 8: The function $V(r)$ versus $r$ for $n=6, p=1.1$, $l=b=1, \Lambda=15, q=1.5$ and $m=0.5$.

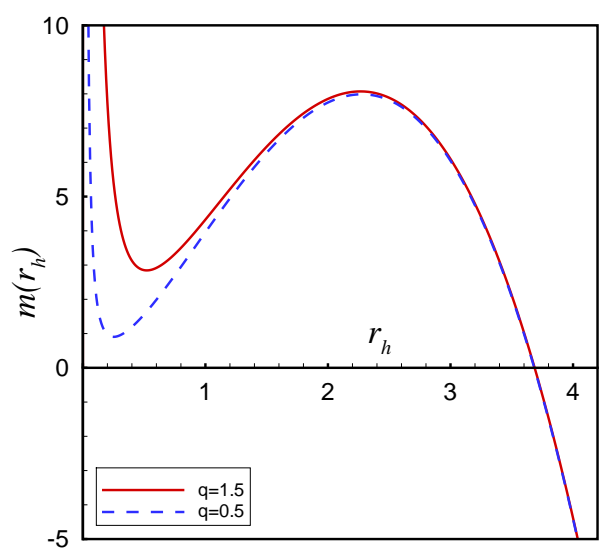

FIG. 9: The function $m\left(r_{h}\right)$ versus $r_{h}$ for $k=1, n=6$, $p=1.1, \alpha=1.18, l=1, b=1.4, \Lambda=-15$.

horizon $r_{+}$can be calculated using the relation

$$
T_{+}=\frac{\kappa}{2 \pi}=\frac{U^{\prime}\left(r_{+}\right)}{4 \pi \sqrt{U / V}}
$$

where $\kappa$ is the surface gravity. One obtains

$$
\begin{aligned}
T_{+}= & \frac{\left(1+\alpha^{2}\right)}{4 \pi}\left\{\frac{k(n-2)}{b^{2 \gamma}\left(1-\alpha^{2}\right) r_{+}^{1-2 \gamma}}-\frac{\Lambda b^{2 \gamma} r_{+}^{1-2 \gamma}}{n-1}\right. \\
& \left.-\frac{2^{p} p(2 p-1) b^{-2(n-2) \gamma p /(2 p-1)} q^{2 p}}{\Pi r_{+}^{(2 p(n-2)(1-\gamma)+1) /(2 p-1)}}\right\} .
\end{aligned}
$$

Comparing the temperature (37) with one calculated in the case of EDPM gravity [22], one finds that temperature is invariant under the conformal transformation (5). This comes from the fact that the conformal parameter at the horizon is regular.

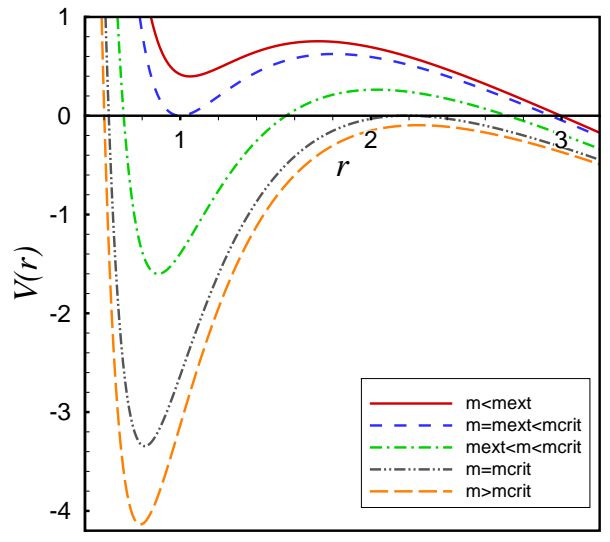

FIG. 10: The function $V(r)$ versus $r$ for $k=1, n=6, p=1.1$, $l=1, b=1.4, \Lambda=-15, \alpha=1.18$ and $r_{\text {ext }}=1$.

The charge of the black hole can be calculated through the modified Gauss law,

$$
Q=\frac{1}{4 \pi} \int(r H)^{n-1}(-F)^{p-1} F_{\mu \nu} n^{\mu} u^{\nu} d \Omega_{n-1},
$$

where $n^{\mu}$ and $u^{\nu}$ are the unit spacelike and timelike normals to a sphere of radius $r$ given as

$n^{\mu}=\frac{1}{\sqrt{-g_{t t}}} d t=\frac{1}{\sqrt{U(r)}} d t, \quad u^{\nu}=\frac{1}{\sqrt{g_{r r}}} d r=\sqrt{V(r)} d r$.

Therefore the charge can be computed as

$$
Q=\frac{2^{p-1} q^{2 p-1} \omega_{n-1}}{4 \pi},
$$

where $\omega_{n-1}$ denotes the volume of constant curvature hypersurface $h_{i j} d x^{i} d x^{j}$. In addition to temperature and charge we need to calculate mass, entropy and electric potential in order to check the satisfaction of the first law of thermodynamics. We can obtain the ArnowittDeser-Misner (ADM) mass $M$, entropy $S$ and electric potential $U$ of the topological black holes by using the Euclidean action method [26]. In this method, one should first fix the electric potential and the temperature on the boundary with a fixed radius $r_{+}$. Then, in order to make the metric positive definite, $t$ should be substituted by $i \tau$ :

$$
d s^{2}=U(r) d \tau^{2}+\frac{1}{V(r)} d r^{2}+r^{2} H^{2}(r) h_{i j} d x^{i} d x^{j} .
$$

This is a necessary step to make the Euclidean action. Since one encounters a conical singularity at the horizon $r=r_{+}$in the Euclidean metric [26], the Euclidean time $\tau$ is made periodic with period $\beta$, where $\beta$ is the inverse of Hawking temperature in order to eliminate this singularity. Now, we are ready to obtain the Euclidean action of $(n+1)$-dimensional BDPM black hole. The 
Euclidean action has two parts namely bulk and surface. The Euclidean action can be calculated analytically and continuously changing of the action (1) to the Euclidean time $\tau$, i.e.,

$$
\begin{aligned}
I_{B D E}= & -\frac{1}{16 \pi} \int_{\mathcal{M}} d^{n+1} x \sqrt{g}\left(\Phi R-\frac{\omega}{\Phi}(\nabla \Phi)^{2}-V(\Phi)\right. \\
& \left.+(-F)^{p}\right)-\frac{1}{8 \pi} \int d^{n} x \sqrt{h} \Phi\left(K-K_{0}\right),
\end{aligned}
$$

where $K_{0}$ is the trace of the extrinsic curvature for the boundary metric $h$ when $q=0$ and $m=0 . K_{0}$ is added in order to normalize the Euclidean action to zero in back- ground [27]. Using the metric (40), one can obtain

$$
\begin{aligned}
R= & -g^{-1 / 2}\left(g^{1 / 2} U^{\prime} V / U\right)^{\prime}-(n-1)\left[2 n V H^{\prime} /(r H)\right. \\
& +2 V H^{\prime \prime} / H+\left(V^{\prime} / r+V^{\prime} H^{\prime} / H\right) \\
& \left.+(n-2)\left(V / r^{2}+H^{\prime 2} V / H^{2}-k /(r H)^{2}\right)\right] \\
K= & -\frac{\sqrt{V}\left[r H U^{\prime}+2(n-1)\left(U H+r U H^{\prime}\right)\right]}{2 r H U},
\end{aligned}
$$

Inserting $U(r)$ and $V(r)$ from (19) and (20) with $q=0$ and $m=0$ in $K$, one obtains

$$
\begin{aligned}
K_{0}= & \left(\frac{b}{r}\right)^{\Gamma / 2}\left(\frac{k(n-2)\left(\alpha^{2}+1\right)^{2} b^{-2 \gamma} r^{2 \gamma}}{\left(\alpha^{2}+n-2\right)\left(\alpha^{2}-1\right)}-\frac{2 \Lambda\left(\alpha^{2}+1\right)^{2} b^{2 \gamma} r^{2-2 \gamma}}{(n-1)\left(\alpha^{2}-n\right)}\right)^{1 / 2} \\
& \left\{2 b^{2 \gamma}\left(\alpha^{2}-1\right)(n \gamma-n+4 p-1+(-6 p+1) \gamma)\left(\alpha^{2}+n-2\right) n \Lambda r^{2(1-\gamma)}\right. \\
& \left.+(n-2)\left(n-\alpha^{2}\right)(n-1) k\left[(n \gamma-n-6 p \gamma+4 p-1)(n-1)-2(p-1) \alpha^{2}\right] r^{2 \gamma} b^{-2 \gamma}\right\} \\
& r^{-1}(n-4 p+1)^{-1}\left[2 b^{2 \gamma} \Lambda\left(\alpha^{2}-1\right)\left(\alpha^{2}+n-2\right) r^{2(1-\gamma)}+r^{2 \gamma} b^{-2 \gamma} k(n-1)(n-2)\left(n-\alpha^{2}\right)\right]^{-1}
\end{aligned}
$$

Substituting $R$ and $K_{0}$ give by Eqs. (42) and (44) in the Euclidean action (41) and using Eqs. (19)-(21), after a long calculation, one can compute the Euclidean action in terms of the model parameters as

$$
\begin{aligned}
I_{B D E}= & \frac{\omega_{n-1}}{4}\left\{\beta \frac{b^{(n-1) \gamma}(n-1) m}{4 \pi\left(1+\alpha^{2}\right)}-\left(b^{(n-1) \gamma} r_{+}^{(n-1)(1-\gamma)}\right)\right. \\
& \left.-\beta \frac{2^{p}(n-1) p^{2} q^{2 p}}{2 \pi b^{-(2 p-n+1) \gamma /(2 p-1)} \Pi \Upsilon r_{+}^{\Upsilon}}\right\} .
\end{aligned}
$$

On the other hand, we know that the thermodynamic potential can be given by $I_{B D E}[27-30]$

$$
I_{B D E}=\beta M-S-\beta U Q,
$$

where $M$ is the ADM mass, $S$ is entropy and $U$ is electric potential. One can easily compare Eq. (45) with Eq. (46) and find that

$$
\begin{gathered}
M=\frac{b^{(n-1) \gamma}(n-1) m \omega_{n-1}}{16 \pi\left(\alpha^{2}+1\right)}, \\
S=\frac{b^{(n-1) \gamma} r_{+}^{(n-1)(1-\gamma)} \omega_{n-1}}{4}, \\
U=\frac{(n-1) p^{2} q}{b^{-(2 p-n+1) \gamma /(2 p-1)} \Pi \Upsilon r_{+}^{\Upsilon}} .
\end{gathered}
$$

Here, it is worthwhile to give some remarks. First, it is notable that the quantities obtained in this section, either conserved or thermodynamic ones, coincide with those calculated in [22]. This fact shows that these quantities are invariant under the conformal transformation (5). It is also worth to note that although entropy is invariant under conformal transformations, it does not follow the area law [31] in contrast with the case of dilaton black holes in the presence of power-law Maxwell field [22]. This is due to the fact that, in the Euclidean action (45) formalism the entropy comes from the boundary term. Now, we turn back to the main purpose of this section which is seeking for satisfaction of first law of thermodynamics for topological $\mathrm{BD}$ black holes. It is a matter of calculations to check that the conserved and thermodynamic quantities calculated in this section satisfy the first law of black hole thermodynamics

$$
d M=T d S+U d Q .
$$

It is also worth mentioning that since the thermodynamic quantities of our topological solutions in BDPM gravity coincide with the ones in EDPM gravity, thermal stability discussions are the same. The stability of the topological black hole under thermal perturbations in EDPM gravity has been discussed extensively in [22].

\section{CLOSING REMARKS}

In this paper, we constructed a new class of topological black hole solutions of BD theory in the presence of a power-law Maxwell field with the Lagrangian. For 
this purpose, we introduced the conformal transformation (5) that transforms the EDPM Lagrangian to the BDPM one. Then, by using this conformal transformation, we obtained BD solutions from Einstein-dilaton solutions presented in [22]. This fact that the BD scalar field is a localized function accompanied with the fact that the effects of mass and charge should disappear at infinity restrict the allowed ranges of the power of electromagnetic source Lagrangian, $p$, and the BD coupling constant, $\omega$. Consequently, we showed that the permitted ranges of $p$ and $\omega$ are $1 / 2<p<(n+1) / 4$ and $\omega>$ $-n /(n-1)+(n-4 p+1)(n+1) / 4 p^{2}(n-1)(n-2)$. In these allowed ranges, our BD solutions are always well defined, except for $\omega=\left((n-4 p+1)^{2}-4 n p^{2}\right) / 4 p^{2}(n-1)$ in the cases of spherical $(k=1)$ and hyperbolic $(k=-1)$ horizons. Also, self-interacting potential $V(\Phi)$ is bounded from below for suitable choices of the model parameters which guarantees the stability of the system. It is worth mentioning that in the above ranges of $p$ and $\omega$, the charge term is always dominant in the vicinity of $r=0$ and positive in metric functions and, therefore, there are no Schwarzschild-like solutions. Next, we studied the conditions under which we have black holes with and without cosmological horizon. Interestingly enough, we showed that our solutions have cosmological horizon even in the presence of negative cosmological constant.

In order to check the satisfaction of the first law of black holes thermodynamics, we first computed temperature and charge. Then, by calculating the Euclidean action, we obtained the black hole's mass, entropy and electromagnetic potential energy. Using these thermodynamic and conserved quantities, we showed that the first law of thermodynamics is satisfied. We found that the entropy does not obey the so-called area law and the conserved and thermodynamic quantities are invariant under conformal transformation (5). All our results recover the results of [15] for linear Maxwell field in the limiting case where $p=1$.

Finally, we would like to mention that in this paper we obtained static topological black hole solutions of BD gravity in the presence of power-law Maxwell nonlinear electrodynamics. For future studies, one can extend the studies to the rotating black holes/branes in BD gravity with power-law Maxwell source. One may also consider other nonlinear electromagnetic sources such as Born-Infeld, logarithmic and exponential Lagrangian and obtain the black hole solutions of these theories in the framework of BD gravity.

\section{Acknowledgments}

We thank Shiraz University Research Council. This work has been supported financially by the Research Institute for Astronomy and Astrophysics of Maragha, Iran.
[1] G. Nordstrom, Relativitatsprinzip und gravitation, Phys. Zeit. 13, 1126 (1912); Träge und schwere masse in der relativitetsmechanik, Ann. Phys. (Berlin) 345, 856 (1913); Zur theorie der gravitation vom standpunkt des relativitetsprinzips, Ann. Phys. (Berlin) 347, 533 (1913); Die fallgesetze und planetenbewegungen in der relativitatstheorie, Ann. Phys. (Berlin) 43, 1101 (1914); A. Einstein and A. D. Fokker, Die Nordströmsche gravitationstheorie vom standpunkt des absoluten differentialkalküls, Ann. Phys. (Berlin) 349, 321 (1914); A. Einstein, Zum gegenwärtigen stande des gravitationsproblems, Phys. Zeit. 14, 1249 (1914).

[2] A. Einstein, Zur allgemeinen relativitätstheorie, Sitz. d. Kon. Preu. Aka. d. Wiss., 778 (1915); Nachtrag zu zur allgemeinen relativitätstheorie, Sitz. d. Kon. Preu. Aka. d. Wiss., 799 (1915); Erklärung der Perihelbewegung des Merkur aus der allgemeinen Relativitätstheorie, Sitz. d. Kon. Preu. Aka. d. Wiss., 831 (1915); Die Feldgleichungen der Gravitation, Sitz. d. Kon. Preu. Aka. d. Wiss. , 844 (1915).

[3] M. Laue, Die Nordströmsche gravitationstheorie, Jahrb. Radioakt. Elektron. 14, 263 (1917); Die Relativitätstheorie (Friedrich Vieweg und Sohn, Braunschweig, Germany, 1921).

[4] A. G. Riess et al., Observational evidence from supernovae for an accelerating universe and a cosmological constant, Astron. J. 116, 1009 (1998); S. Perlmutter et al., Measurements of omega and lambda from 42 high- redshift supernovae, Astrophys. J. 517, 565(1999); J. L. Tonry et al., Cosmological results from high-z supernovae, Astrophys. J. 594, 1 (2003); A. T. Lee et al., A high spatial resolution analysis of the MAXIMA-1 cosmic microwave background anisotropy data, Astrophys. J. 561, L1 (2001); C. B. Nettereld et al., A measurement by BOOMERANG of multiple peaks in the angular power spectrum of the cosmic microwave background, Astrophys. J. 571, 604 (2002); N. W. Halverson et al., DASI first results: A measurement of the cosmic microwave background angular power spectrum, Astrophys. J. 568, 38 (2002); D. N. Spergel et al., First Year Wilkinson Microwave Anisotropy Probe (WMAP) observations: Determination of cosmological parameters, Astrophys. J. Suppl. 148, 175 (2003).

[5] C. Brans and R. H. Dicke, Mach's principle and a relativistic theory of gravitation, Phys. Rev. 124, 925 (1961).

[6] C. H. Brans, The roots of scalar-tensor theory: An approximate history, arXiv:gr-qc/0506063.

[7] Th. P. Sotiriou and V. Faraoni, $f(R)$ theories of gravity, Rev. Mod. Phys. 82, 451 (2010).

[8] I. Zh. Stefanov, S. S. Yazadjiev, and M. D. Todorov, Scalar-tensor black holes coupled to Born-Infeld nonlinear electrodynamics, Phys. Rev. D 75, 084036 (2007); I. Zh. Stefanov, S. S. Yazadjiev, and M. D. Todorov, Scalartensor black holes coupled to Euler-Heisenberg nonlinear electrodynamics, Mod. Phys. Lett. A 22, 1217 (2007); I. Zh. Stefanov, S. S. Yazadjiev, and M. D. Todorov, Phases 
of 4D scalar-tensor black holes coupled to Born-Infeld nonlinear electrodynamics, Mod. Phys. Lett. A 23, 2915 (2008).

[9] C. H. Brans, Mach's principle and a relativistic theory of gravitation. II, Phys. Rev. 125, 2194 (1962).

[10] A. Bhadra and K. Sarkar, On static spherically symmetric solutions of the vacuum Brans-Dicke theory, Gen. Relativ. Gravit. 37, 2189 (2005).

[11] R. G. Cai and Y. S. Myung, Black holes in the BransDicke-Maxwell theory, Phys. Rev. D. 56, 3466 (1997).

[12] M. H. Dehghani and M. Shojania, Travelling-wave-type and stationary soliton solutions of the Brans-Dicke equation, Can. J. Phys. 80, 951 (2002).

[13] M. H. Dehghani, J. Pakravan, and S. H. Hendi, Thermodynamics of charged rotating black branes in Brans-Dicke theory with quadratic scalar field potential, Phys. Rev. D 74, 104014 (2006).

[14] A. Sheykhi and M. M. Yazdanpanah, Thermodynamics of charged Brans-Dicke AdS black holes, Phys. Lett. B 679, 311 (2009).

[15] A. Sheykhi and H. Alavirad, Topological Black Holes in Brans-Dicke-Maxwell Theory, Int. J. Mod. Phys. D 18, 1773 (2009).

[16] M. Born and L. Infeld, Foundations of the new field theory, Proc. R. Soc. A 144, 425 (1934).

[17] H. H. Soleng, Charged black points in general relativity coupled to the logarithmic $U(1)$ gauge theory, Phys. Rev. D 52, 6178 (1995).

[18] S. H. Hendi, Asymptotic charged BTZ black hole solutions, J. High Energy Phys. 03, 065 (2012); S. H. Hendi and A. Sheykhi, Charge rotating black string in gravitating nonlinear electromagnetic fields, Phys. Rev D 88, 044044 (2013).

[19] A. Sheykhi and N. Riazi, Thermodynamics of black holes in $(n+1)$-dimensional Einstein-Born-Infeld dilaton gravity, Phys. Rev. D 75, 024021 (2007); A. Sheykhi, Topological Born-Infeld-dilaton black holes, Phys. Lett. B 662, 7 (2008); A. Sheykhi, Thermodynamical properties of topological Born-Infeld-dilaton black holes, Int. J. Mod. Phys. D 18, 25 (2009); A. Sheykhi and S. Hajkhalili, Dilaton black holes coupled to nonlinear electrodynamic field, Phys. Rev. D 89, 104019 (2014); A. Sheykhi and A. Kazemi, Higher dimensional dilaton black holes in the presence of exponential nonlinear electrodynamics, Phys. Rev. D 90, 044028 (2014); A. Sheykhi, F. Naeimipour, and S. M. Zebarjad, Phase transition and thermodynamic geometry of topological dilaton black holes in gravitating logarithmic nonlinear electrodynamics, Phys. Rev. D 91, 124057 (2015).

[20] M. Hassaine and C. Martinez, Higher-dimensional black holes with a conformally invariant Maxwell source, Phys. Rev. D 75, 027502 (2007).

[21] M. Hassaine and C. Martinez, Higher-dimensional charged black holes solutions with a nonlinear electrodynamics source, Classical Quantum Gravity 25, 195023 (2008); H. Maeda, M. Hassaine, and C. Martinez, Lovelock black holes with a nonlinear Maxwell field, Phys.
Rev. D 79, 044012 (2009); H. A. Gonzalez, M. Hassaine, and C. Martinez, Thermodynamics of charged black holes with a nonlinear electrodynamics source, Phys. Rev. D 80, 104008 (2009); S. H. Hendi and B. Eslam Panah, Thermodynamics of rotating black branes in Gauss-Bonnet-nonlinear Maxwell gravity, Phys. Lett. B 684, 77 (2010); S. H. Hendi, Rotating black branes in the presence of nonlinear electromagnetic field, Eur. Phys. J. C 69, 281 (2010); A. Bazrafshan, M. H. Dehghani and M. Ghanaatian, Surface terms of quartic quasitopological gravity and thermodynamics of nonlinear charged rotating black branes, Phys. Rev. D 86, 104043 (2012); S. H. Hendi and M. H. Vahidinia, Extended phase space thermodynamics and P-V criticality of black holes with nonlinear source, Phys. Rev. D 88, 084045 (2013); M. H. Dehghani, A. Sheykhi, and S. E. Sadati, Thermodynamics of nonlinear charged Lifshitz black branes with hyperscaling violation, Phys. Rev. D 91, 124073 (2015); M. Kord Zangeneh, A. Sheykhi, and M. H. Dehghani, Thermodynamics of topological nonlinear charged Lifshitz black holes, Phys. Rev. D 92, 024050 (2015); M. Kord Zangeneh, A. Sheykhi, and M. H. Dehghani, Thermodynamics of charged rotating dilaton black branes with powerlaw Maxwell field, Eur. Phys. J. C 75, 497 (2015).

[22] M. Kord Zangeneh, A. Sheykhi, and M. H. Dehghani, Thermodynamics of higher dimensional topological dilation black holes with a power-law Maxwell field, Phys. Rev. D 91, 044035 (2015).

[23] D. R. Brill, J. Louko, and P. Peldan, Thermodynamics of $(3+1)$-dimensional black holes with toroidal or higher genus horizons, Phys. Rev. D 56, 3600 (1997).

[24] R. G. Cai and K. S. Soh, Topological black holes in the dimensionally continued gravity, Phys. Rev. D 59, 044013 (1999).

[25] C. S. Peca and J. P. S. Lemos, Thermodynamics of Reissner-Nordstrom-anti-de Sitter black holes in the grand canonical ensemble, Phys. Rev. D 59, 124007 (1999); C. S. Peca and J. P. S. Lemos, Thermodynamics of toroidal black holes, J. Math. Phys. 41, 4783 (2000).

[26] R. G. Cai, R. K. Su and P. K. N. Yu, Thermodynamics for black strings and p-branes, Phys. Lett. A 195, 307 (1994).

[27] J. D. Brown, E. A. Martinez, and J. W. York Jr., Complex Kerr-Newman Geometry and Black Hole Thermodynamics, Phys. Rev. Lett. 66, 2281 (1991).

[28] H.W. Braden, J. D. Brown, B. F. Whiting and J.W. York Jr., Charged black hole in a grand canonical ensemble, Phys. Rev. D 42, 3376 (1990).

[29] B. F. Whiting and J. W. York Jr., Action Principle and Partition Function for the Gravitational Field in Black Hole Topologies, Phys. Rev. Lett. 61, 1336 (1988).

[30] J.W. York Jr., Black-hole thermodynamics and the Euclidean Einstein action, Phys. Rev. D 33, 2092 (1986).

[31] G. Kang, Black hole area in Brans-Dicke theory, Phys. Rev. D 54, 7483 (1996). 